\title{
Toward a Physical Characterization of Raindrop Collision Outcome Regimes
}

\author{
F. Y. TESTIK \\ Civil Engineering Department, College of Engineering and Science, Clemson University, Clemson, South Carolina \\ A. P. BARROS \\ Civil and Environmental Engineering Department, Pratt School of Engineering, Duke University, Durham, North Carolina \\ L. F. BLIVEN \\ NASA GSFC Wallops Flight Facility, Wallops Island, Virginia
}

(Manuscript received 18 October 2010, in final form 18 December 2010)

\begin{abstract}
A comprehensive raindrop collision outcome regime diagram that delineates the physical conditions associated with the outcome regimes (i.e., bounce, coalescence, and different breakup types) of binary raindrop collisions is proposed. The proposed diagram builds on a theoretical regime diagram defined in the phase space of collision Weber numbers We and the drop diameter ratio $p$ by including critical angle of impact considerations. In this study, the theoretical regime diagram is first evaluated against a comprehensive dataset for drop collision experiments representative of raindrop collisions in nature. Subsequently, the theoretical regime diagram is modified to explicitly describe the dominant regimes of raindrop interactions in (We, $p$ ) by delineating the physical conditions necessary for the occurrence of distinct types of collision-induced breakup (neck/filament, sheet, disk, and crown breakups) based on critical angle of impact consideration. Crown breakup is a subtype of disk breakup for lower collision kinetic energy that presents distinctive morphology. Finally, the experimental results are analyzed in the context of the comprehensive collision regime diagram, and conditional probabilities that can be used in the parameterization of breakup kernels in stochastic models of raindrop dynamics are provided.
\end{abstract}

\section{Introduction}

The objective of this paper is to introduce a comprehensive regime diagram that delineates the physical conditions associated with the outcome regimes (i.e., bounce, coalescence, and different breakup types) of binary raindrop collisions. The work builds on a theoretical regime diagram proposed by Testik (2009) to distinguish coalescence and breakup regions in the phase space of drop collision Weber number We and drop diameter ratio $p$ based on laboratory observations of water drop collisions at the bench scale. The theoretical regime diagram is first evaluated against experimental data from tower-based measurements of drop

Corresponding author address: Dr. Firat Y. Testik, 110 Lowry Hall, Civil Engineering Department, Clemson University, Clemson, SC 29634-0911.

E-mail: ftestik@clemson.edu collisions similar to natural rainfall, including continuous production of raindrops at terminal velocity (Barros et al. 2008). Next, the data from Barros et al. are used to modify the regime diagram to describe explicitly the raindrop breakup regime diagrams that are critical to capture the dynamical evolution of rain drop size distributions (DSDs) in microphysical models (e.g., Prat and Barros 2007a, 2009).

The competing roles of coalescence (accretion) and breakup in the dynamical evolution of warm rain DSDs were hypothesized as early as 1948 by Langmuir (Langmuir 1948). Drop coalescence is a consequence of drop collisions alone, whereas drop breakup may be caused by three distinct mechanisms: drop collisions, aerodynamic instability, and electrostatic forces. The relative importance of each of these mechanisms in governing drop breakup occurrences and ultimately shaping the raindrop size distributions observed in nature has long been a subject of debate. Lack of accurate 


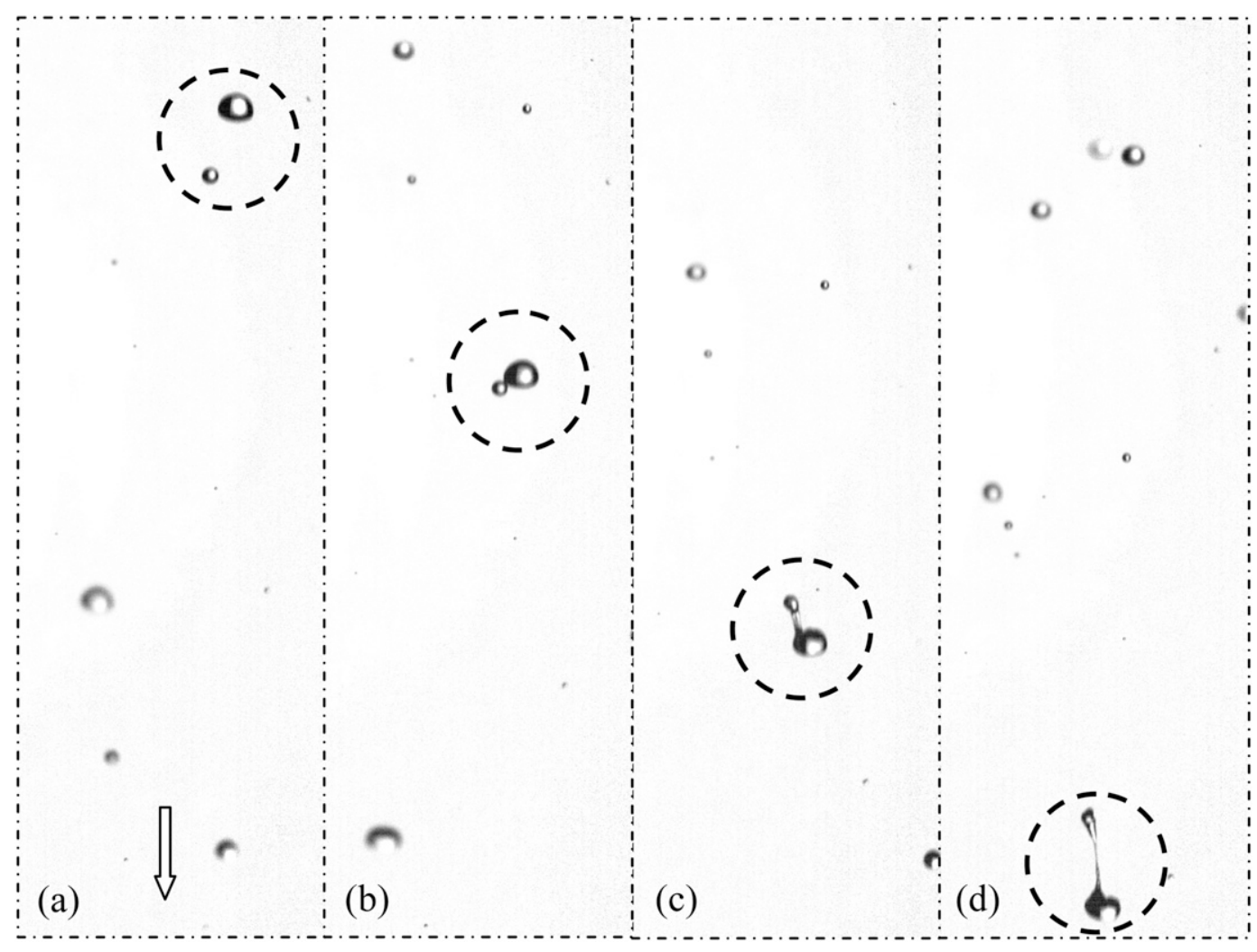

FIG. 1. Neck/filament type of breakup shown by a sequence of high-speed camera images. Arrow indicates the direction of the gravitational acceleration and dashed circles are used to highlight the interacting drops. Vertical and horizontal sizes of each frame are 8.5 and $4 \mathrm{~cm}$, respectively, and provide a length scale.

quantitative information on the amplitudes of the electric fields required for electrostatic drop disruption and the question of whether typical electrical fields in thunderclouds are sufficient to cause such disruptions (see the discussion given by Jones et al. 2010) leave drop collision and aerodynamic instability mechanisms at the center of this debate [see exchange regarding Villermaux and Bossa (2009) and Barros et al. (2010)]. Comprehensive experiments from List and Whelpdale (1969), List et al. (1970), and Low and List (1982a) to Barros et al. (2008) show that drop collisions play a major role in shaping the raindrop size distribution through coalescence and breakup processes, whether the aerodynamic instability mechanism has a sizable contribution.

Prat and Barros (2009) showed the importance of DSD transients to estimate rainfall rates for various storm regimes and conditions. Currently, microphysical models of raindrop dynamics simulate the stochastic evolution of drop populations relying on heuristic parameterizations of the probability of raindrop collision and raindrop collision outcome, that is, no change, coalescence, and breakup type based on relative frequencies determined from tower experiments (e.g., Low and
List 1982b). Besides the limited duration of such experiments, and therefore limited sample size, accurate imaging of drop collisions involving very small drops (typically $<0.5 \mathrm{~mm}$ ) is difficult, or even impossible, at the resolution required with available technology (Barros et al. 2008). For example, Prat and Barros (2010b) showed that, in order to capture the observed rainfall intensities from tropical storm systems consistent with characteristics of observed DSDs (Tokay et al. 2008), the number of drops with diameter $<0.1 \mathrm{~mm}$ was one order of magnitude higher than commonly used. Therefore, the dynamics of small raindrops, specifically in the submillimeter range, cannot be ignored. The theoretical (We, $p$ ) regime diagram provides a path to a physically based parameterization of the probability of drop collision outcomes encompassing the full range of drop diameters in natural rainfall.

This manuscript is organized as follows. In section 2, relevant literature in raindrop collision research is reviewed. In section 3, the laboratory experimental setup and data analysis are described. In section 4, the theoretical regime diagram for drop collision outcomes developed by Testik (2009) is evaluated with the dataset collected at the National Aeronautics and Space 


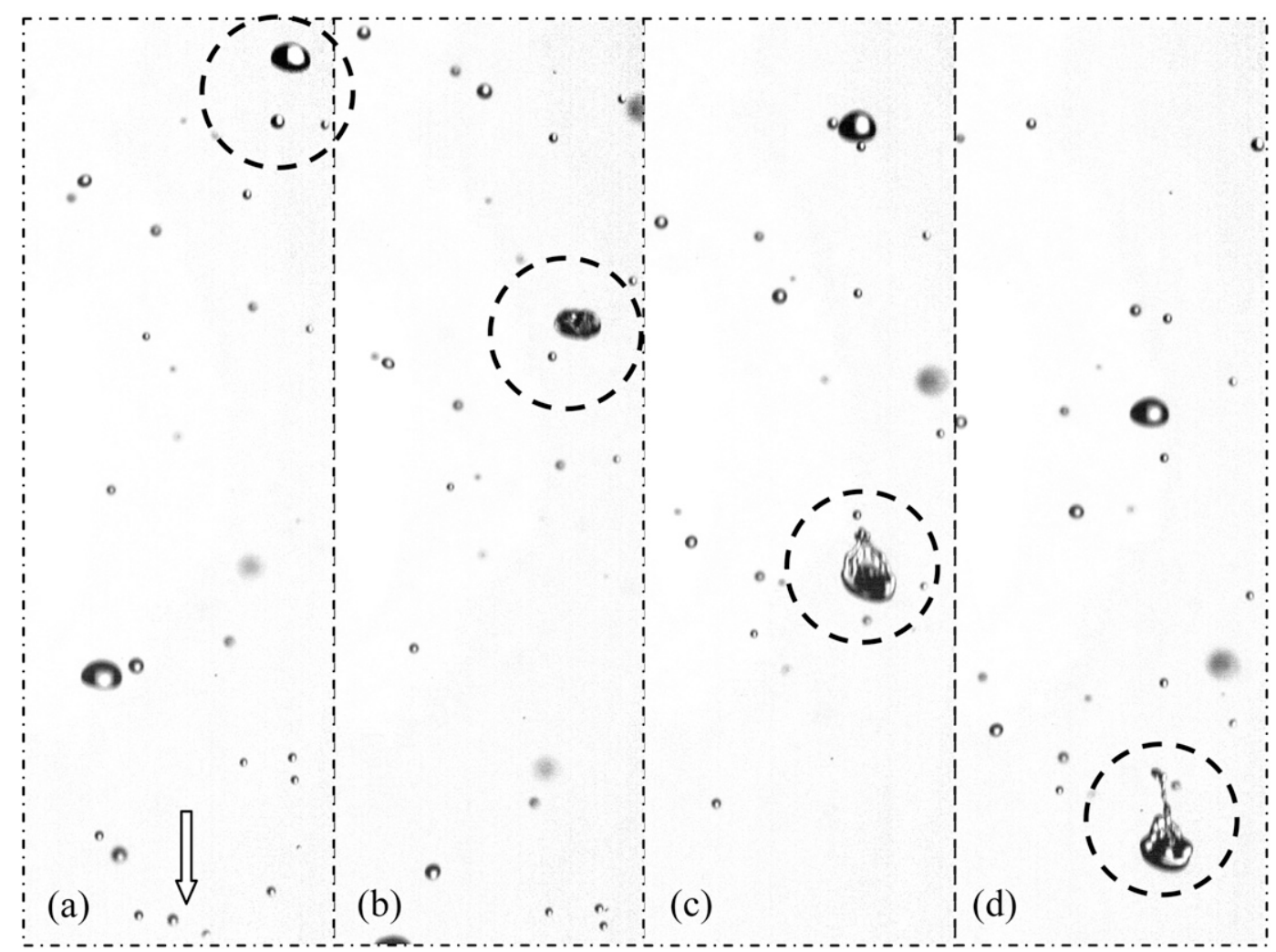

FIG. 2. Sheet type of breakup shown by a sequence of high-speed camera images. Vertical and horizontal sizes of each frame are 8.5 and $4 \mathrm{~cm}$, respectively, and provide a length scale.

Administration Wallops Flight Facility (WFF), and the diagram is further extended to provide a more comprehensive diagram that includes also subregimes (i.e., breakup patterns) of collision outcomes. Discussion and conclusions are given in section 5 .

\section{Review of raindrop collision studies}

\section{a. Breakup regimes}

Collisions of two raindrops may yield three different collision outcome scenarios/regimes: colliding drops may bounce apart, coalesce, or break up. McTaggartCowan and List (1975) classified collision-induced drop breakups into four different categories: neck (also referred to as filament), sheet, disk, and bag breakups. Photographic presentations of these breakup patterns are detailed in the literature (e.g., Testik and Barros 2007; Testik and Young 2008; Barros et al. 2008; Testik 2009). Because drop breakup is a key aspect of this study, a brief qualitative description of the dynamic evolution of breakup process supported with sequential high-speed images $4 \mathrm{~ms}$ apart is given below to ensure the completeness of this communication. Note that, in addition to the four different drop breakup patterns in the classical literature, a distinct type of disk breakup pattern, which henceforth will be referred to as crown breakup, was observed in the experiments reported here that is relevant not only from a drop morphology point of view but also from the point of view of collision regime, as shown later. Bag breakup was observed only once in our experiments and is not included in our analysis, consistent with the rarity of this breakup type [e.g., $0.5 \%$ of collisions reported by McTaggart-Cowan and List (1975)]. Qualitative observations for each breakup pattern are as follows.

Neck/filament breakup results from a glancing contact that results in the formation of a water neck/bridge that joins the two drops. In Fig. 1, the dynamical evolution of neck breakup is illustrated by sequential high-speed images. As can be seen from these images, the small drop does not appear to affect the large drop except in the immediate vicinity of the point of contact. Upon separation, while the large and small drops are still substantially intact, additional smaller drops (i.e., fragments) form as a result of the dissolution of the water neck/filament.

The sheet breakup pattern is presented by sequential high-speed images in Fig. 2. This type of breakup occurs 


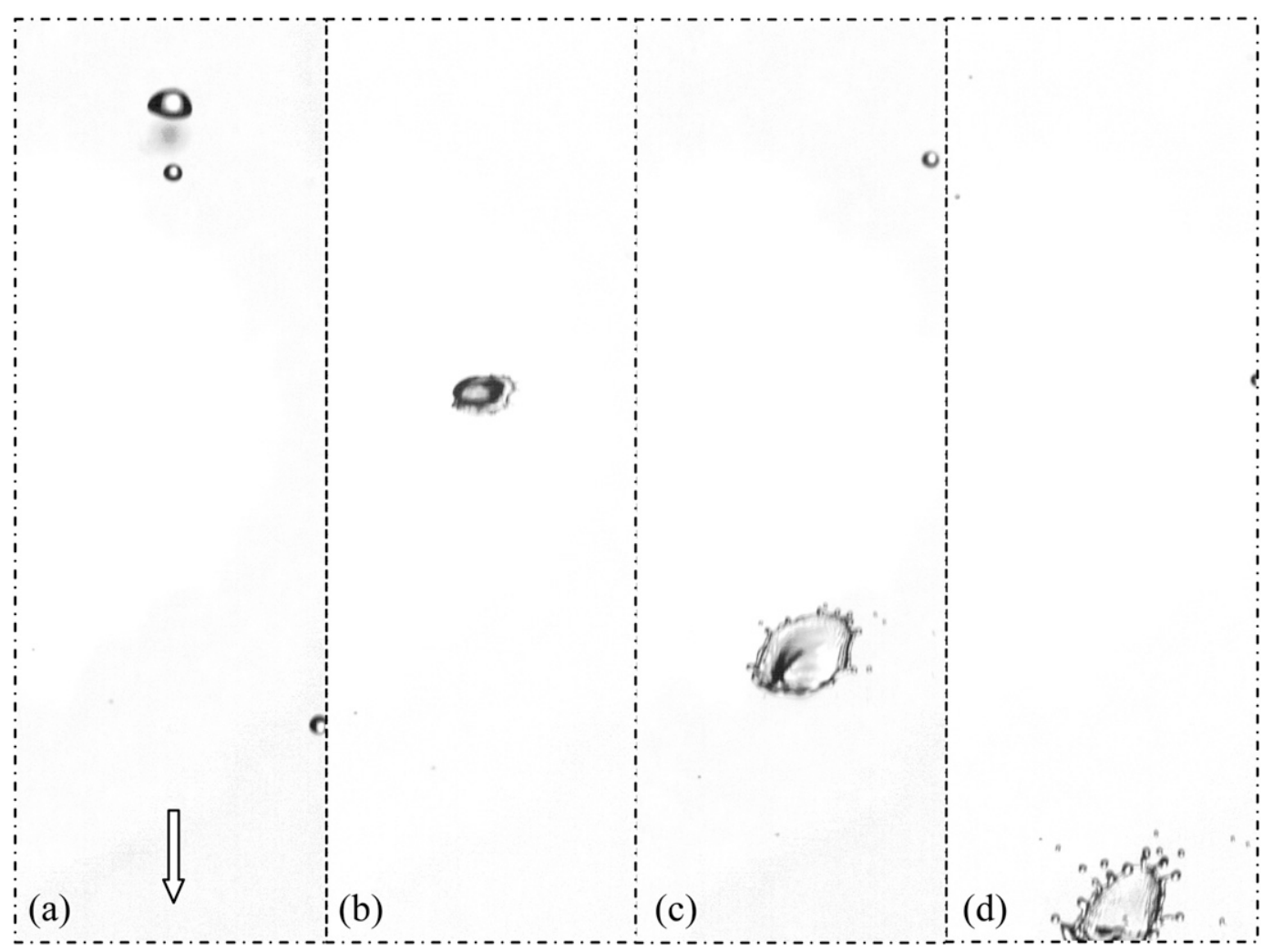

FIG. 3. As in Fig. 2, but for disk type of breakup.

when the larger drop hits the smaller one in such a position that part of the larger drop is torn off, forming an extending film or sheet of water from the impact area. The smaller drop often disappears in this sheet and the larger drop becomes strongly distorted. A number of fragments result from the disintegration of this sheet. Compared to neck breakup, sheet breakup occurs when the point of collision takes place closer to the center of the larger drop and it requires more energy induced by the collision.

The disk breakup pattern occurs when the smaller drop hits the larger one near the center, as can be seen in Fig. 3. The two drops temporarily coalesce following the collision and a disk of water begins to extend from the point of impact where the small drop becomes incorporated. During this process, increased drag force acting on the disk-shaped water body causes a rapid deceleration. Once the disk reaches its maximum extent, the outer fringes shed drops and the entire disk gradually disintegrates into a relatively large number of fragments.

Crown breakup, a breakup pattern identified for the first time in this study, corresponds to a subset of disk breakup with similar dynamical evolution but with distinctive morphology and lower collision kinetic energy requirements. Moreover, qualitative observations (visual inspection of frames) indicate that the crown breakup fragment size distribution (FSD) is characterized by larger and fewer drops than the disk breakup fragment size distribution. This is an important distinction for modeling the dynamical evolution of DSDs, in particular coalescence processes that are highly sensitive to the number of small drops. However, a definitive quantitative differentiation of the two for modeling purposes requires image analysis with higher sensitivity (at higher resolution). As shown in Fig. 4, although colliding drops temporarily coalesce (Figs. 4a,b) since collision-induced energy overcomes the surface tension forces, an outward (perpendicular to fall direction) water motion at the point of impact begins to occur. The aerodynamic forces, which try to restore a near-spherical drop shape, resist the flattening of the drop shape. As a result, the water motion starts curling (opposite to the direction of the gravity vector) and the distorted drop displays a bowl shape with "beaded fringes" (Figs. 4c,d). This instability results in shedding of the smaller drops from the fringe tips, followed by fringe collapse leading to the formation of a large, severely distorted drop. The general mechanism for the fragmentation at the fringe tips is the same for the crown and disk breakups. In both breakups, a free rim at the edge of the liquid sheet forms by surface tension (see Yarin 2006). This rim forms cusps, a usual process when 


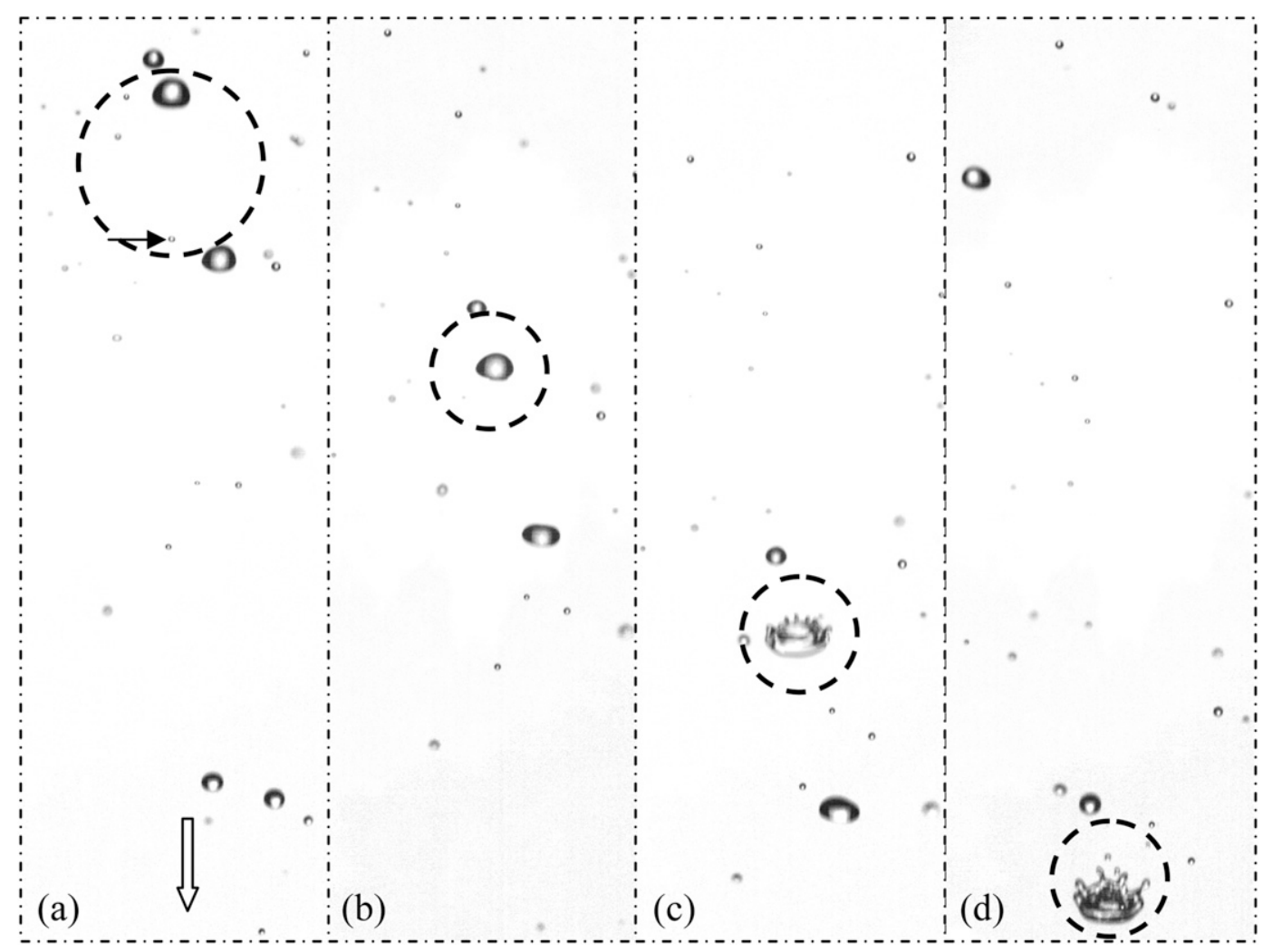

FIG. 4. As in Fig. 2, but for crown type of breakup. This type of breakup is documented for the first time in this study.

the rim is disturbed (Yarin 1993). At the cusps, water jets are discharged, which form the beaded fringes and then fragmentation occurs due to capillary breakup.

\section{b. Previous work}

The overarching goal in studying colliding raindrops is to quantitatively determine the outcome regimes of drop collisions (i.e., bounce, coalescence, and different breakup types) and the number and size of forming fragments. This present study is concerned only with the outcome regimes of raindrop collisions. Therefore, major advances related mainly to this aspect of raindrop collision studies are discussed in this section. Also note that studies on collisions of cloud droplets $(<0.1 \mathrm{~mm}$ in diameter) are not our focus; hence are not discussed.

Controlled laboratory experimentation has been the main approach in studying raindrop collisions owing to the difficulties in theoretical and numerical treatment of this complex three-dimensional, two-phase flow problem. Adequate laboratory simulations of raindrop collisions have a number of inherent challenges, and these challenges have dictated the development of raindrop collision experiments since the mid-twentieth century. Earlier laboratory studies attempted to investigate raindrop collisions using two different types of simplified setups. The first setup is the vertical wind tunnel (e.g., Blanchard 1948, 1949; Montgomery 1971) in which the larger of the colliding drops is suspended aerodynamically and the smaller one is released from below to set off a collision. The second setup may be referred to as a drop-support system in which a small drop is directed at a larger drop that is suspended from the tip of a capillary tube or a hypodermic needle (e.g., Magano and Nakamura 1959; List and Whelpdale 1969). The relative simplicity of these setups compromised an important criterion for adequate simulations of natural rainfall conditions: establishing the correct terminal velocities of colliding drops in the direction of gravity. This criterion was first met by Magarvey and Geldart (1962) in their raindrop collision experiments under free fall conditions. Given the large fall distances required for satisfying this criterion ( $\sim 8-12 \mathrm{~m}$ depending on the drop diameter-see Beard 1977; Wang and Pruppacher 1977) and associated experimental challenges-adoption of free fall experiments have been gradual. First, free fall experiments were conducted without giving consideration to the terminal velocity of the colliding drops (i.e., nonterminal drops; e.g., Gunn 1965; Brazier-Smith et al. 1972). Then, List et al. (1970) relaxed the criterion from establishing the correct terminal velocities for each colliding drop to 
establishing the correct differential fall velocities between the colliding drops, which was sufficient for correct simulation of the collision kinetic energy (CKE),

$$
\mathrm{CKE}=\left(\frac{\rho \pi}{12}\right) \frac{d_{1}^{3} d_{2}^{3}}{d_{1}^{3}+d_{2}^{3}} \Delta V^{2},
$$

where $\Delta V=\left|\mathbf{V}_{1}-\mathbf{V}_{2}\right|$ is the absolute value of the speed of the colliding drops relative to each other, $d$ the drop diameter (i.e., the diameter of a sphere having the same volume as the drop), $\mathbf{V}$ the drop velocity, and $\rho$ the density of the liquid; subscripts 1 and 2 indicate the larger and smaller of the colliding drops, respectively, throughout the text. Finally, McTaggart-Cowan and List (1975), emphasizing the importance of correct absolute velocities of the colliding drops for adequate simulation of the fluid dynamics processes of collisions, set the standard for raindrop collision experiments by fully satisfying the terminal free fall criterion.

There are a variety of factors determining the fate of raindrop collisions: velocities and sizes of the colliding drops, angle of impact, surface tension, electric charges, ambient pressure, temperature, humidity, and others (List and Whelpdale 1969; Park 1970). Moreover, raindrops may oscillate (see Andsager et al. 1999; Testik et al. 2006) and collisions of oscillating drops may have different outcomes than the collisions of equilibriumshaped raindrops. A physically meaningful quantitative depiction of raindrop collision outcomes involving all these governing factors has not been available due to obvious difficulties. Simplifications in the factors considered and a systematic approach are inevitable. The basic combination of factors that one needs to include in studying raindrop collision outcomes includes drop velocities and sizes, angle of impact, and surface tension. This combination of factors leads to three governing dimensionless parameters: We $\left[=\rho\left(d_{2} / 2\right)(\Delta V)^{2} / \sigma\right.$ in which $\sigma$ is the surface tension of the liquid], $p\left(=d_{2} / d_{1}\right)$, and impact angle $\theta$; see definition sketch in Fig. 5. Knowledge gained through such simplified studies would serve as the base to build upon with the findings of the studies investigating the effects of the environmental/ ambient factors. A deterministic approach to quantify raindrop collision outcomes based on the governing dimensionless parameters has been a difficult endeavor. Multiple raindrop collision outcomes for fixed experimental conditions, rather than only a single outcome as in a deterministic problem, were often reported. Ochs et al. (1995) argued that this may be due to experimental uncertainty and that the determinism hypothesis is likely to hold true as evidenced by broad regions of collision outcomes separated by sharp borders reported by, for example, Park (1970). This is a very likely explanation, given that experimental measurement errors (e.g., impact angle location) may be considerable and control of the experimental conditions (e.g., surface tension value, electrical charges) are challenging (e.g., Menchaca-Rocha et al. 1997).

Low and List (1982a,b), adding onto the experimental measurements by McTaggart-Cowan and List (1975), generated an experimental database of raindrop collision outcomes. They investigated collisions of 10 different drop pairs, with over 100 collisions for each pair. Based on their experimental observations, Low and List proposed an empirical equation involving CKE and surface tension energy $\left(\mathrm{SE}=\pi \sigma d^{2}\right)$ to estimate the probability of coalescence occurrence. They also incorporated an energy condition involving CKE and surface energies with an experimentally defined cutoff value to demarcate the physical conditions for which coalescence is expected to be absent. Since their experiments with high CKE values did not reveal any bounce observations, overall breakup occurrence probability is parameterized by simply subtracting the coalescence occurrence probability parameterization from unity. Moreover, occurrence probabilities of each observed breakup type (filament/neck, sheet, and disk) are empirically parameterized using CKE and surface energies. This study has been the building block for numerical simulations of raindrop size distribution evolution studies over the last three decades (e.g., List and McFarquhar 1990; McFarquhar 2004; Prat and Barros 2007a,b).

Barros et al. (2008) conducted experiments, similar to Low and List (1982a), focusing on the evaluation of fragment size distributions (FSDs) from different types of breakup. The experimental FSDs for the collision of selected drop pairs were evaluated against explicit simulations using a dynamical microphysics model. The model was found to underestimate the fragment numbers observed in the smallest diameter range (e.g., $D<$ $0.2 \mathrm{~mm}$ ) and to overestimate the number of fragments produced when the colliding drop pairs are such that the smaller of the colliding drops is larger than or equal to $1 \mathrm{~mm}$ in diameter and the larger of the colliding drops is larger than or equal to $3 \mathrm{~mm}$ in diameter. This effect was particularly large for fragments in the $0.5-1.0-\mathrm{mm}$ range, and more so for filament breakup (the most frequent type of breakup observed in laboratory conditions), reflecting up to $30 \%$ uncertainty in the left-hand side of the FSD (i.e., the submillimeter range). Their experiments point out the need for further experimentation to elucidate the physics of breakup regimes for very small raindrops.

Recently, Schlottke et al. (2010), and Straub et al. (2010) in the accompanying study, extended the raindrop collision outcome database formed by Low and List 

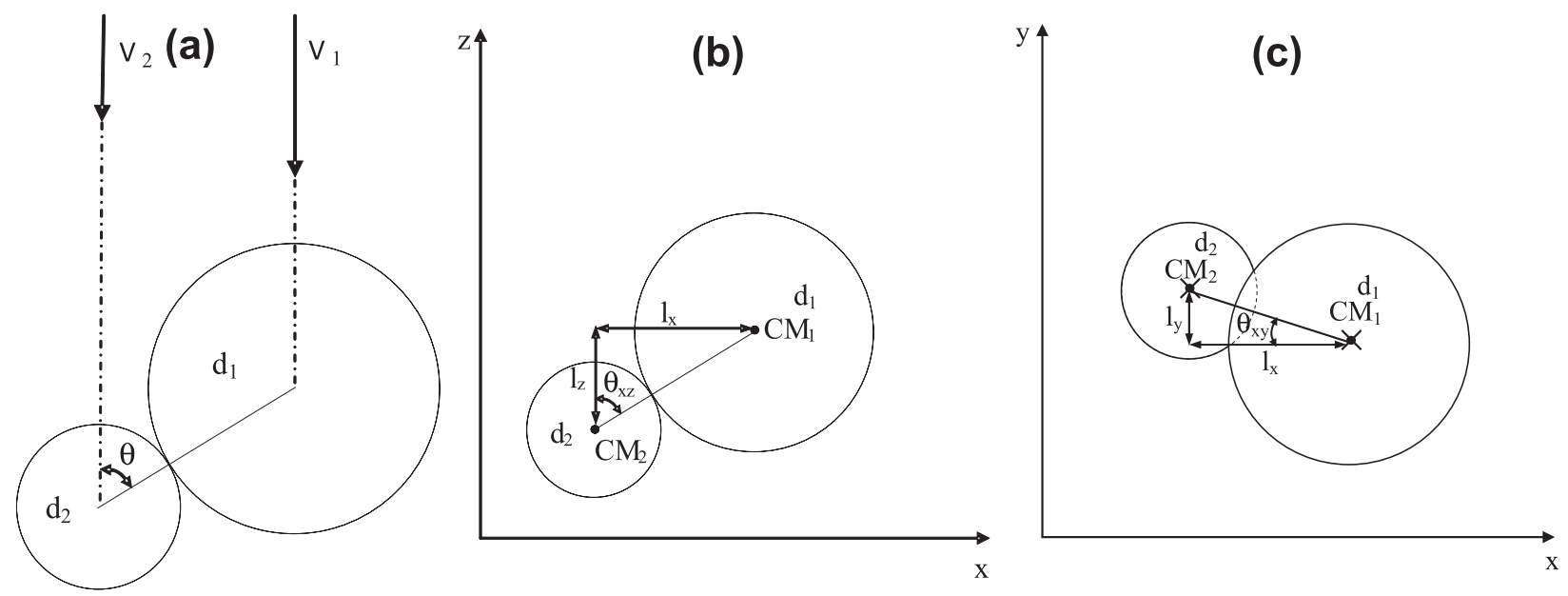

FIG. 5. (a) Simplified 2D schematic of the impact geometry for drops traveling in the same direction (such as raindrops falling vertically: negative $z$ direction). Impact angle calculations require the values of the three components of the distance vector $\left(l_{x}, l_{y}, l_{z}\right)$ between the center of masses (CM) of the two drops, which can be obtained by (b),(c) two orthogonal views. Raindrop fall velocity, diameter, and impact angle are denoted by $V, d$, and $\theta$, respectively. Subscripts 1 (2) denote the larger (smaller) of the colliding drops and the subscripts $x, y$, and $z$ denote the respective coordinate axes; $\theta_{x z}$ and $\theta_{x y}$ denote the angle of impact components in the $x-z$ and $x-y$ planes as shown in the schematic. This figure is reproduced from Testik (2009).

(1982a,b) through direct numerical simulation (DNS) of collisions of 32 drop pairs with sizes chosen to cover nearly completely the entire size parameter range relevant to breakup after Low and List (1982b) and Barros et al. (2008). Results of their numerical simulations deviated from the experimental findings of Low and List (1982a,b). In particular, coalescence occurrence probabilities were different and a new empirical parameterization for coalescence occurrence probability involving again CKE and surface energies was proposed. Moreover, the existence of a cutoff value of CKE necessary for coalescence proposed by Low and List (1982a,b) could not be confirmed. It is important to note, however, that Barros et al. (2008) confirmed the drop collision energy cutoff (ET) estimated by Low and List (i.e., ET $>5.0 \mu \mathrm{J}$ ). Nevertheless, as pointed out by Straub et al. (2010), the limitation of the DNS-based parameterizations (Schlottke et al. 2010; Straub et al. 2010) is that they do not take into account all of the governing physical factors; the limitation of experiment-based parameterizations is that results are limited by the measurement technology and experimental constraints. This includes impact angle or a similar geometric impact parameter (e.g., eccentricity, see Straub et al. 2010) that is one of the constituents of the basic combination of factors as discussed above.

Parameterizations that include all three constituents of the basic combination of factors have been attempted mainly by constructing two-parameter regime diagrams for constant values of the third parameter. Such an early attempt by Brazier-Smith et al. (1972) resulted in collision outcome regime diagrams in the Weber number-geometric impact parameter plane for fixed values of colliding drop diameter ratios. In this diagram, regime delineations are based on the relative importance of rotational kinetic and surface energies. This study was conducted for drop collisions with large angles between velocity vectors of the drops. Low and List (1982a) pointed out that rotational kinetic energy is not a parameter of primary importance for raindrop collisions as such oblique drop collisions do not adequately simulate raindrop collisions in nature. Schlottke et al. (2010) noted that rotational kinetic energy is at least one order of magnitude smaller than CKE for raindrop collisions. Ashgriz and Poo (1990) proposed new drop collision outcome regime diagrams again in the Weber numbergeometric impact parameter plane for fixed values of colliding drop diameter ratios. Their experiments involved drop collisions with large angles between the velocity vectors of the drops as in Brazier-Smith et al. (1972). However, based on their experimental observations they note that breakup occurs much earlier than the development of any significant rotation. Hence, they developed regime separation parameterizations based on energy balance arguments between linear kinetic and surface energies rather than rotational kinetic and surface energies as in Brazier-Smith et al. (1972). Recently, Testik (2009) developed a regime diagram in the Weber number-drop diameter ratio plane. In this diagram, regime separation parameterizations are based on the relative importance of CKE and surface energies as discussed in the next section separately. There are other important studies aimed at development of drop collision outcome 
regime diagrams based on different physical arguments (e.g., Adam et al. 1968; Park 1970; Qian and Law 1997). owing to space constraints these studies are not discussed here.

\section{c. Drop collision outcome regime diagram}

The regime diagram proposed by Testik (2009) [henceforth called the T09 diagram, see Fig. 6] is the building block for the theoretical component of this study. To ensure the autonomy of this present study and for the convenience of the reader, a detailed overview of the T09 diagram parameterizations is given in this section. The reader is kindly referred to Testik (2009) for detailed physical discussions.

The T09 diagram lies in the Weber number-drop diameter ratio (We-p) plane. In this diagram (Fig. 6), the physical conditions that are responsible for the occurrence of collision outcome regimes are delineated based on two competing energies: the surface energy of the drop that acts to maintain drop integrity and the collision kinetic energy that has a destabilizing effect. Note that Testik neglected the effects of viscous losses, an assumption employed by many researchers in similar raindrop studies (e.g., Brazier-Smith et al. 1972; Ashgriz and Poo 1990; Villermaux and Bossa 2009). This assumption can be justified based on the Ohnesorge parameter

$$
\mathrm{Oh}=\frac{16 \mu}{(\rho R \sigma)^{1 / 2}}
$$

in which $R$ is the drop radius and $\mu$ the dynamic viscosityrange arguments for raindrops composed of relatively low viscosity water (see Ashgriz and Poo 1990; Testik 2009). Indeed, Gotaas et al. (2007) showed that viscous dissipation losses during formation of the drop collision outcome regimes are small for low viscosity fluids, such as water. Based on their numerical simulations, Schlottke et al. (2010) reported that viscous dissipation losses may be important only after the raindrop collision is completed (i.e., after the drop collision outcome regimes formed). In the T09 diagram, two separation curves demarcate the $\mathrm{We}-p$ plane into three regions (marked as I, II, and III in Fig. 6). Region I delineates the conditions in which coalescence and neck/filament-type breakup occurs. Region II delineates the conditions in which only breakup (in the absence of bounce and coalescence) occurs, and region III delineates the conditions in which only bounce (in the absence of coalescence and breakup) occurs.

The regime separation curves in the T09 diagram are defined as follows [see Testik (2009) for physical reasoning]:

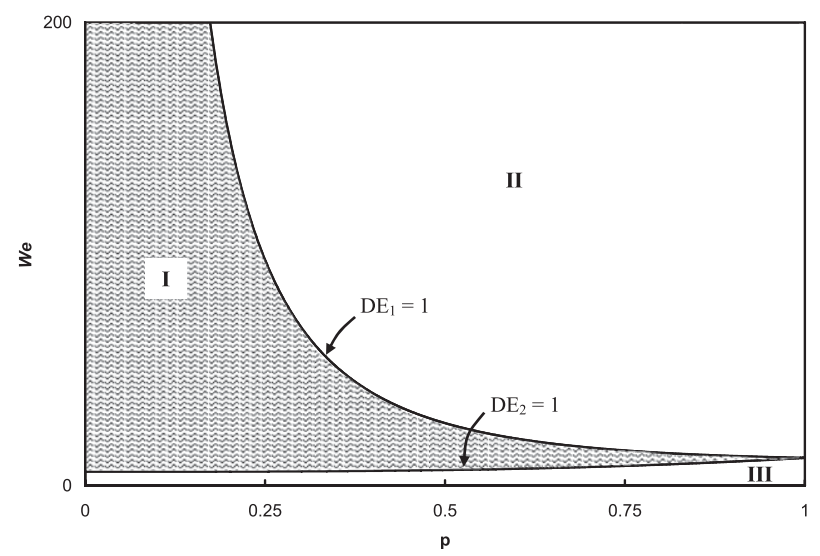

FIG. 6. The theoretical regime diagram is divided into three regions according to two regime separation curves (solid lines). Each region delineates the physical conditions for the occurrence of a different regime: coalescence and neck/filament type breakup (region I), breakup (region II), and bounce (region III).This figure is reproduced from Testik (2009).

$$
\begin{aligned}
& \mathrm{DE}_{1}=\frac{\mathrm{CKE}}{\mathrm{SE}_{1}}=\frac{p^{2} \mathrm{We}}{6\left(1+p^{3}\right)}=1, \\
& \mathrm{DE}_{2}=\frac{\mathrm{CKE}}{\mathrm{SE}_{2}}=\frac{\mathrm{We}}{6\left(1+p^{3}\right)}=1 .
\end{aligned}
$$

Here DE represents the dimensionless energy based on the ratio of CKE and SE of the colliding drops. Corresponding regime separation curves are shown in Fig. 6.

The T09 diagram does not involve impact angle, leaving out the third constituent of the basic combination of factors for drop collisions discussed above. The hypothesis was that the impact angle is not an important governing parameter for characterizing the conditions for the occurrence of main collision outcome regimes (i.e., bounce, coalescence, and breakup) except in the case of coalescence and neck/filament breakup regimes in region I of the regime diagram (near-grazing collisions lead to neck breakup and nearly head-on collisions lead to coalescence in region I). However, the impact angle is an important governing parameter to delineate the conditions for both the occurrence of different breakup types in region II and for coalescence and neck breakup in region I of the regime diagram. The experimental data showed a strong support to this hypothesis, but experimental measurement limitations (i.e., lack of impact angle measurements with sufficient accuracy) did not permit delineation of impact angle conditions for the occurrence of different breakup types. In addition, the bench-scale experiments in Testik (2009) did not replicate fundamental aspects of natural rainfall such as collisions between drops at terminal velocity. Incorporating this dependency is essential for numerical 
simulations of raindrop size distribution evolution and is one goal of the present study.

\section{Experimental setup}

Results from the drop collision experiments presented in this manuscript were conducted at the Rain-Sea Interaction Laboratory at NASA WFF and were first described and analyzed by Barros et al. (2008). Here, a more detailed description of the experimental setup is provided to document how similarity with natural rainfall was achieved. The NASA WFF laboratory houses an indoor rain tower $17-\mathrm{m}$ high with a base area $4 \times 4 \mathrm{~m}^{2}$ [see Bliven and Elfouhaily (1993) for details of this facility]. A ladder at one corner of the tower leads to the catwalk platform located $14 \mathrm{~m}$ above the ground. This platform is used to locate drop generators at the top of the rain tower. In the experiments, two streams of drops are generated; one from the top of the rain tower (at the catwalk platform) and one from the top of a pole, $5 \mathrm{~m}$ above the camera level (see Fig. 7). In the experiments, two streams of drops with different sizes are generated; one with the larger drops from the top of the rain tower (at the catwalk platform) and one with the smaller drops from the top of the 5-m pole (see Fig. 7). To distinguish between these two drop sources henceforth, the top of the rain tower is simply referred as the "ceiling" and drops generated from the ceiling as "large drops." Similarly, the top of the side pole is known as the "pole" and drops generated from the pole are called "small drops." To avoid any confusion arising from the use of this terminology, refer to the schematic in Fig. 7 to visualize the experimental configuration. Raindrop collisions are recorded using a high-speed camera capable of recording up to 1000 frames per second and a maximum resolution of $1280 \times 1024$ pixels.

In the experiments, tap water was filtered by two filters connected in series. The first filter collects the particulates with diameters larger than $30 \mu \mathrm{m}$, and the second collects the particulates with diameters larger than $5 \mu \mathrm{m}$. Surface tension measurements of filtered water indicated approximately (within $\pm 3 \%$ ) the surface tension value for clean water $\left(\sigma=7.28 \times 10^{-2} \mathrm{~N} \mathrm{~m}^{-1}\right)$. Therefore, the surface tension value for clean water is used in calculations throughout this study. Two identical pumps (Cole-Parmer Masterflex L/S) supply filtered water with precisely controlled flow rates. One pump is connected to two manifolds of six outlets each that are connected to an array of 12 hypodermic needles (set up in two rows with each needle separated by $1 \mathrm{~cm}$ ) of the same tip diameter placed at the ceiling. The other pump is connected to a single hypodermic needle attached to the pole.

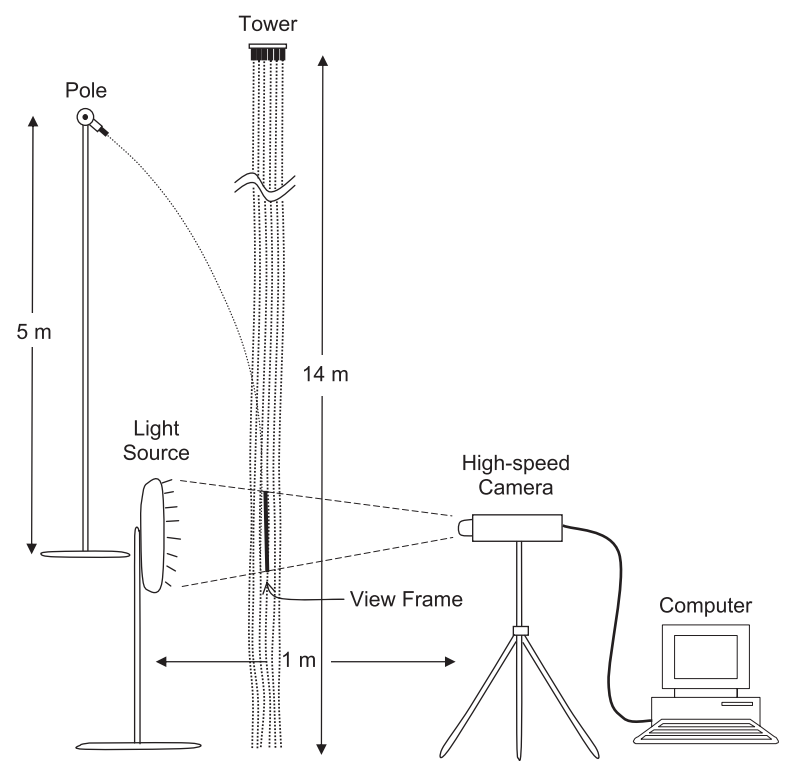

FIG. 7. Schematic of the experimental setup at the NASA WFF rain laboratory. Drops generated from a platform $14 \mathrm{~m}$ above ground level at the tower and from a pole $5 \mathrm{~m}$ above camera level collide at the view frame of the high-speed camera. The camera points to the light source, standing $1 \mathrm{~m}$ apart; captured collision images are transferred to a computer via RS-232 cable.

Six different flat-tip needle size combinations are used to generate drops with different sizes (diameters) from the ceiling and pole. Histograms showing the occurrence percent for diameters of colliding (i.e., parent) drops are given in Fig. 8. Here, black bars in Fig. 8a indicate the large drops from the ceiling and textured bars in Fig. 8b indicate the small drops from the pole. Large drops are generated by dripping water at the needle tips while small drops are generated by the breakup of the water jet formed by pumping pressurized water through the single needle attached to the pole. This experimental technique allowed us to generate collisions of drops with a broad size spectrum (see Fig. 8) consistent with observed rain DSDs. The main goal of this study is to delineate the physical conditions associated with the distinct raindrop collision outcome regimes independently of the drop size distribution, and thus independently of their actual frequency of occurrence in natural conditions, which is controlled by the DSD.

A critical specification of the experimental design was to produce collisions of drops with steady-state shapes (i.e., nonoscillating equilibrium shapes) that fall at terminal velocities in the same direction as the gravity vector, similar to raindrop collisions in natural rainfall. However, note that collisions between oscillating drops to the extent they happen are included in the experimental dataset and are therefore parameterized in terms 

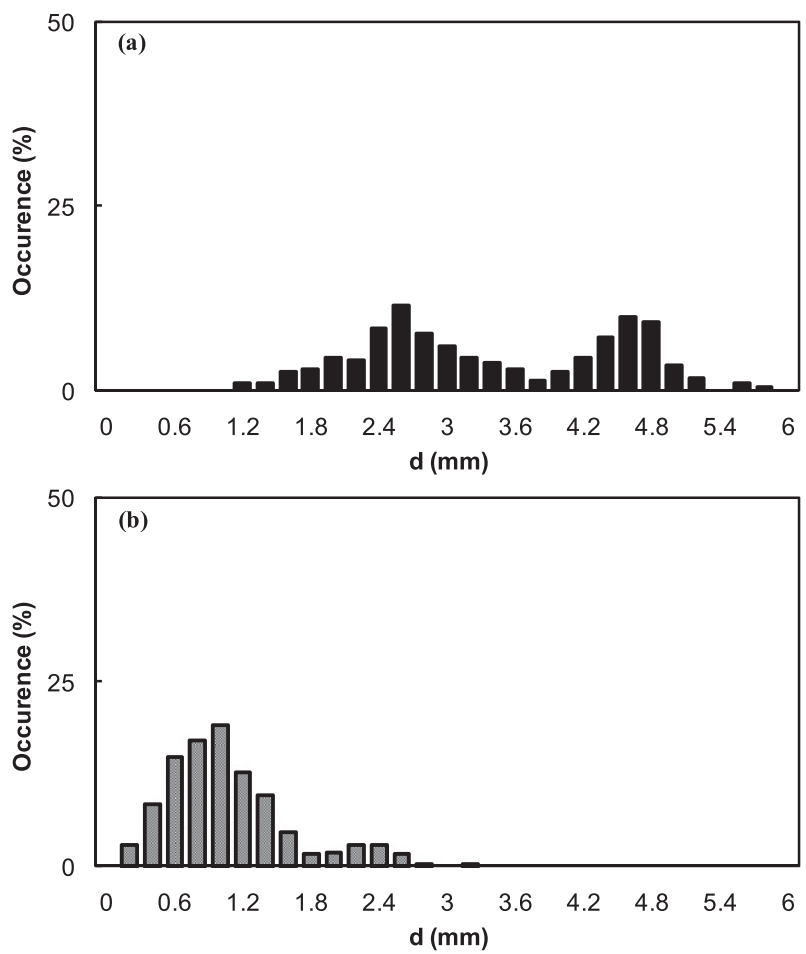

FIG. 8. Histograms showing the occurrence percentage (ordinate) of the parent drop diameters (abscissa): (a) large drops generated from the ceiling (black bars) and (b) small drops generated from the pole (textured bars).

of their equilibrium shape and the simplified energy balance model (i.e., proposed regime diagram). Such a drop collision configuration (i) allows inference of the maximum angle of impact values using a single high-speed camera rather than using two synchronized orthogonal high-speed cameras (see Testik 2009), (ii) allows coverage of a larger We number range, and (iii) eliminates possible unsteady effects from oscillations and acceleration/ deceleration of the drops. The height differentials between the ceiling and pole and the control volume monitored by the high-speed camera is sufficient to reach terminal velocity for the ranges of drop sizes measured here (Wang and Pruppacher 1977). Visual inspection of the movies indicates that the vertical fall distance for oscillating drops near the generation source was sufficient to reach steadystate shapes. A comparison of the vertical fall velocities $V$ of colliding drops measured just before collisions and estimated terminal fall velocities $V_{t}$ from a well-accepted parameterization by Lhermitte (1990) given in Fig. 9 indicates that generated drops from the ceiling have enough time to reach terminal velocities before collisions. The comparison also shows that drops generated from the pole have enough time to relax to near-terminal fall velocities (slightly larger terminal fall velocity values than estimations; i.e., superterminal velocities) after the breakup of

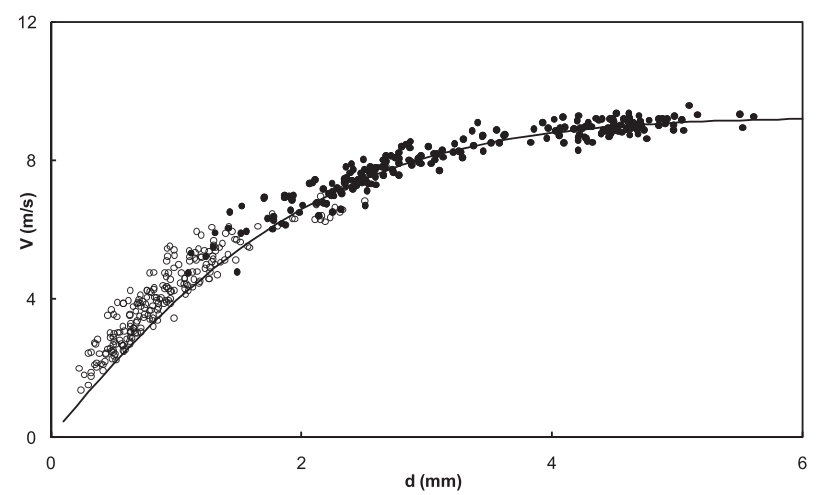

FIG. 9. Comparison of the vertical fall velocities of parent drops measured for large drops (solid circles) and small drops (open circles) just before the collisions and the estimations of terminal fall velocities (solid line) by Lhermitte (1990).

jet. As sketched in Fig. 7, the projectile of small drops is fine-tuned (by rotating the needle and changing the flow rate) such that at the camera view frame, where collisions are aimed to occur, the motion of the small drop is mainly vertical (parallel to the gravity vector) and lateral drop motion is minimal. Although for clarity the pole is drawn in the schematic as if standing behind the light source, the actual placement of the pole is such that the water jet is generated parallel to the camera view plane. As shown in Fig. 10, lateral fall speeds $U$ of the drops parallel to the camera view plane are approximately $7 \%$ (see the solid line in the figure) of vertical fall speeds. Note that percent values of lateral to vertical fall speed ratios of both large and small drops prior to collisions are presented in this figure (same drops as in Fig. 9). Here, although small drops generated from the pole display lateral fall speeds relative to vertical fall speeds that are slightly more pronounced than large drops from the ceiling, this small difference shows the effect of the initial lateral direction of the water jet velocity to be insignificant. This is important because the lateral velocity should be much smaller than the differential vertical velocity of the drop pair so that the collision dynamics is determined primarily by the differential vertical velocity just before impact. Lateral drift observations with larger amplitudes $(20 \%-30 \%$ of vertical fall speeds) were reported by Testik et al. (2006) for oscillating raindrops observed in natural rain. Lateral fall speeds of the large drops observed in the present laboratory experiments are instantaneous and the direction of lateral drop motion changes continuously. The resulting lateral displacement of large drops reaching the experimental measurement area is bounded in a circle of radius approximately $10 \mathrm{~cm}$ centered at the initial point of drop generation. Observed lateral drift of nonoscillating large drops in the absence of external forcing mechanisms (such as wind forcing) in this controlled laboratory study 


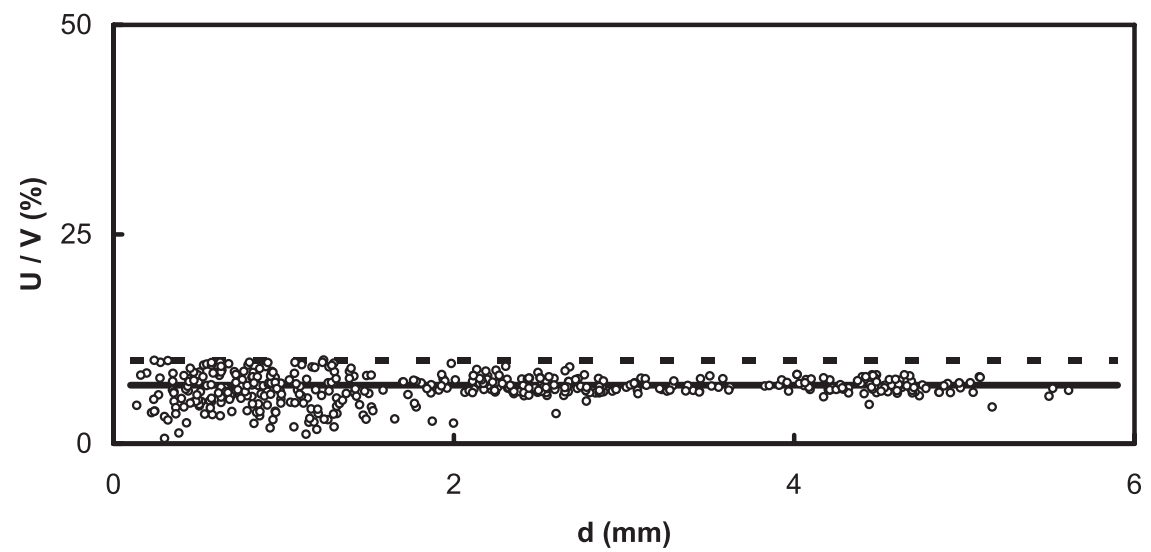

FIG. 10. Percentage of the lateral $(U)$ to vertical $(V)$ fall velocities of parent drops (large and small drops) as a function of drop diameter. Open circles indicate measurements; solid and dashed lines indicate $7 \%$ (mean value for the measurements) and $10 \%$ (maximum value for the measurements) values, respectively.

is due to the inherent vortex shedding mechanism in the drop wake [see discussions in Testik and Barros (2007) and Testik et al. (2006)]. Lateral displacements of the small drops are fine-tuned for binding within a depth of approximately $1.5-2 \mathrm{~cm}$ perpendicular to the camera focal plane. This adjustment allows for measurement accuracy considerations for the drop imaging and analysis technique used. Tests with calibrated glass spheres showed that measurement accuracy of the drop characteristics for the described experimental configuration is bounded to approximately $12 \%$. In the experiments, camera lens settings are adjusted to record images with a pixel size that corresponds to $\sim 0.1 \mathrm{~mm} \times 0.1 \mathrm{~mm}$. Details of the drop imaging technique in the experiments were described by Testik et al. (2006). An image processing algorithm in LabVIEW environment was developed to enhance and process these acquired collision images. This algorithm is used to measure important precollision characteristics of colliding drops, such as drop diameter, fall velocity, angle of impact, and others. Note that a measured drop diameter corresponds to the diameter of an equivalent disk (i.e., diameter of the disk with the same area as the recorded drop image). A total of 322 drop collisions including bounce (6), coalescence (66), and breakup (250: 151 neck, 56 sheet, and 43 disk, 26 of which are crown) are considered in this manuscript. The experimental range of dimensionless parameters covered is $\mathrm{We}=2.5-193.7, p=$ $0.04-0.88$, and $\theta=0.3^{\circ}-82.1^{\circ}$.

\section{Raindrop collision outcome regime diagram}

In this section, the original T09 diagram is first evaluated using the raindrop experimental observations at NASA WFF. Next, the theoretical regime diagram is modified to delineate the regions of the $(\mathrm{We}, p)$ space corresponding to each identified raindrop breakup type based on experimental observations and critical angle of impact $\theta_{\mathrm{cr}}$, and energy considerations as discussed below.

The critical angle of impact values characterizing the separations between different regimes are experimentally deduced here from single camera measurements. Although orthogonal precollision images are required for measurements of correct impact angles (through measurement of the distance vector components shown in Figs. 5b and 5c), critical impact angles may be estimated from single camera measurements as follows. The angle of impact measurements by a single camera are based on the projections of colliding drops to the camera view frame (see Fig. 5a); these angle values may be smaller than or equal to (when the collision plane is parallel to the camera view frame) the correct $\theta$. Therefore, for single camera measurements one expects to observe a scatter of measured $\theta$ values starting from zero degrees (when the collision plane-the plane that goes through the center of masses of both of the colliding drops-is perpendicular to the camera view frame) to a maximum value characteristic of the specific regime (see Figs. 11a-f). If large numbers of collision data are collected to ensure that a sufficient number of collisions occurs with collision planes nearly parallel to the camera view frame, then this maximum value (henceforth, upper bound) may be used to estimate the critical value of the impact angle, above which the occurrence of the specific regime is not expected. This procedure assumes that the upper bound is independent of $\mathrm{We}$ and $p$ [i.e., $\theta_{\mathrm{cr}} \neq f(\mathrm{We}, p), \theta_{\mathrm{cr}}$ is a constant]. Indeed, the experimental observations indicate no noticeable functional dependency of $\theta_{\mathrm{cr}}$ on We and $p$, supporting this assumption. Although occurrence of some regimes may also be bounded by a minimum impact 

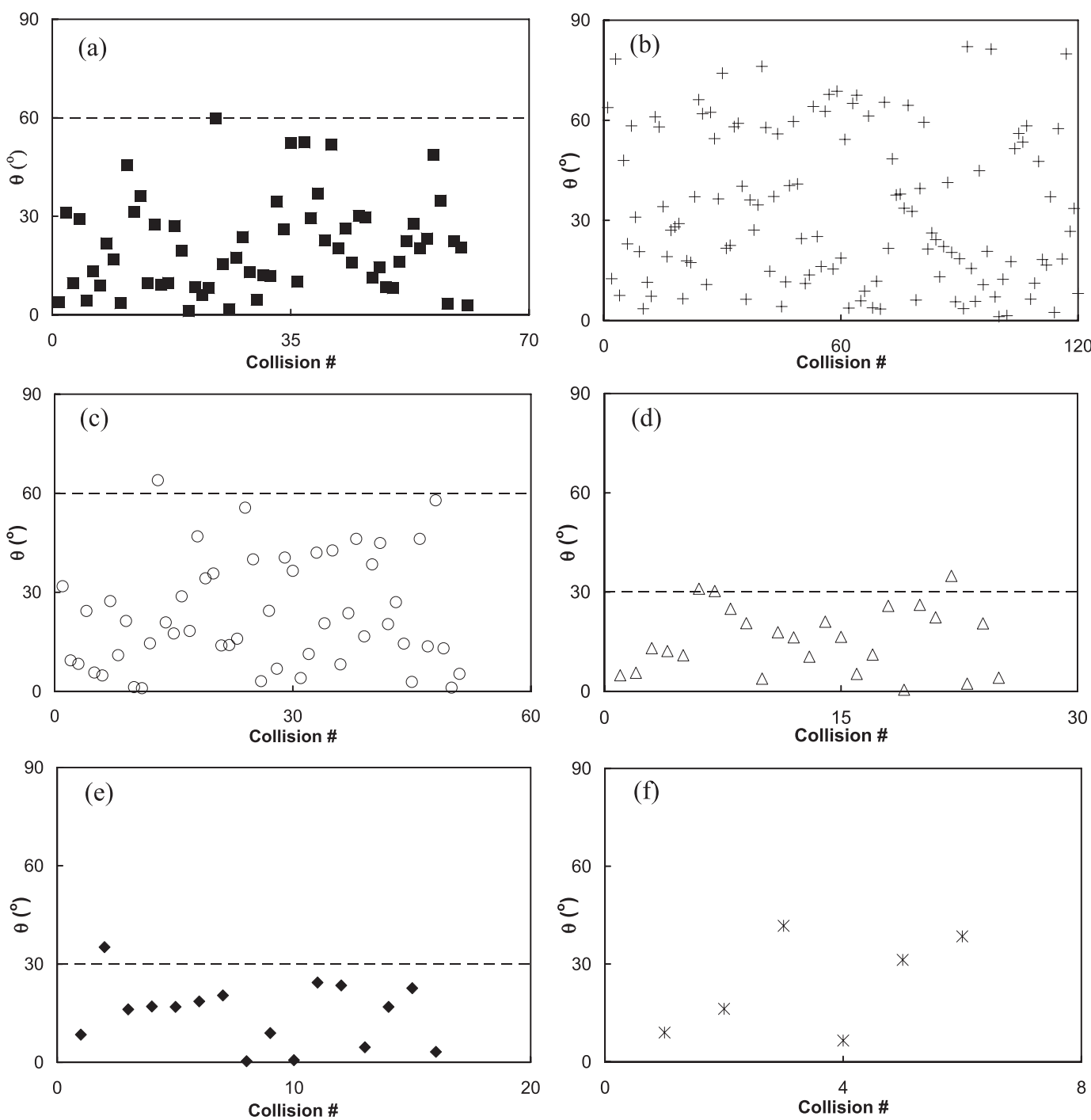

FIG. 11. Impact angle measurements based on the projections of colliding drops to the camera view frame vs a dummy collision count index for different regimes: (a) coalescence, (b) neck, (c) sheet, (d) crown, (e) disk, and (f) bounce. Symbols represent experimental observations and dashed lines represent upper bounds of impact angle for each regime.

angle value (henceforth, the lower bound), single camera measurements do not allow extraction of information on such minimum impact angle values. However, the lower bound for a specific regime may be estimated as the upper bound of another regime within the same region of the We- $p$ plane. This consideration implies the assumption that physical conditions leading to two different regimes cannot overlap in the We- $p-\theta$ space.

Figure 11 shows scatterplots of measured $\theta$ values for each regime. From the plots, one can approximate upper bounds for coalescence and three of the breakup regimes as $60^{\circ}$ for coalescence (Fig. 11a) and sheet breakup (Fig. 11c) and $30^{\circ}$ for crown (Fig. 11d) and disk breakups (Fig. 11e). Consistent with the qualitative description of neck/ filament breakup, $\theta$ measurements given in Fig. 11b do not indicate the existence of an upper bound for neck breakup. Hence, neck breakup may be expected for $\theta$ values up to $90^{\circ}$. A small number of bounce observations shown in Fig. $11 \mathrm{f}$ does not allow identification of an upper bound; however, because bounce is the only regime that occurs in region III of the We- $p$ plane (see Figs. 6 and 12), a bounding constraint in terms of $\theta$ is not expected for bounce.

The regime diagram including all identified subregimes and experimental data collected at NASA WFF is given in Fig. 12. In this diagram, an experimental separation curve corresponding to $\mathrm{DE}_{1}=2$ (the dashed curve in the diagram) is also shown in conjunction with the arguments on separation between the crown and disk 


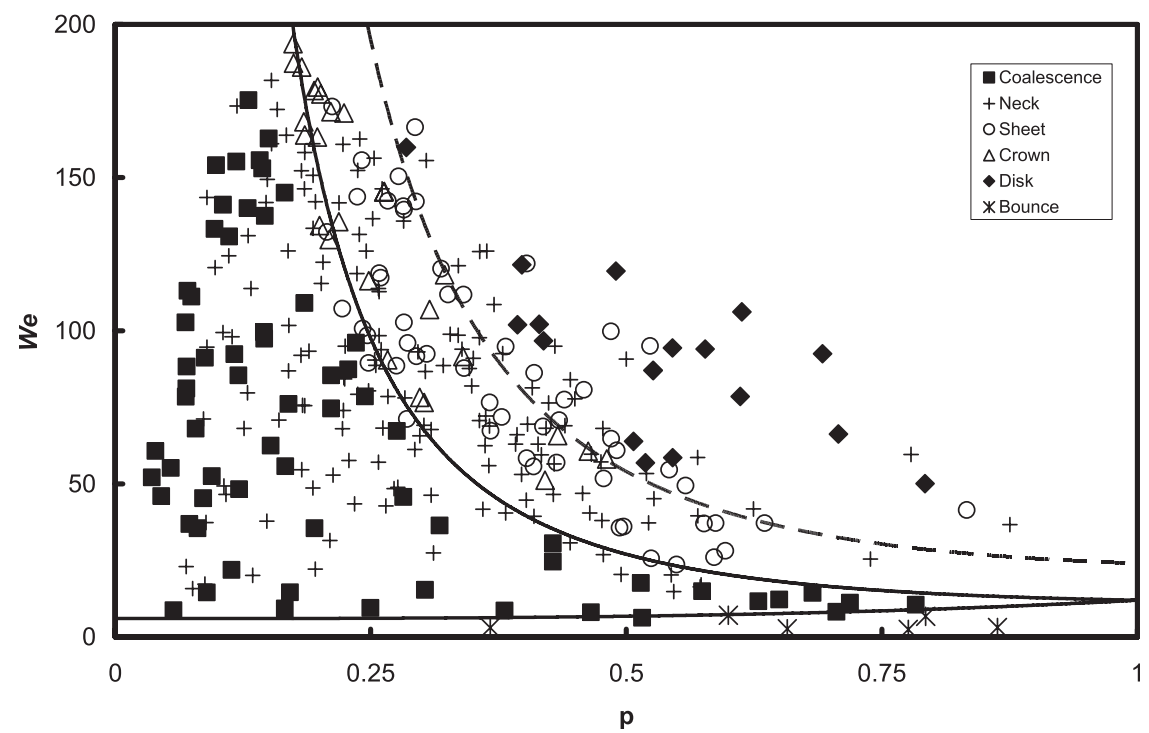

FIG. 12. Regime diagram in the We-p plane by Testik (2009) with experimental drop collision observations at the NASA WFF rain laboratory. Symbols represent experimental observations of different regimes (see the legend) and solid $\left(\mathrm{DE}_{1}=1\right.$ and $\left.\mathrm{DE}_{2}=1\right)$ and dashed $\left(\mathrm{DE}_{1}=2\right)$ lines represent regime separation curves.

breakup regimes given below. Figure 12 clearly confirms the occurrence of coalescence only in region I. Neck/ filament breakup is observed in both regions I and II, whereas sheet and disk breakups are bounded by region II only. Crown and disk breakups are observed in different parts of region II as separated by the $\mathrm{DE}_{1}=2$ curve. Crown breakup is observed in the region bounded by $1<\mathrm{DE}_{1}<2$ (henceforth, region IIa), whereas disk breakup is observed exclusively in the remaining portion of region II ( $\mathrm{DE}_{1}>2$; henceforth, region IIb). This particular value of $\mathrm{DE}_{1}=2$ is an empirical approximation to provide a clear separation between the crown and disk breakup regions in the regime diagram based on the available experimental observations. This value may be subject to slight modifications as new observations become available. As expected from theoretical arguments, bounce is observed only in region III in the absence of any other regimes in this region.

There is overlap of breakup regimes in the diagram (see Fig. 12): coalescence and neck breakup overlap in region I, and neck and sheet breakups overlap with crown and disk breakups in different sectors of region II. However, as mentioned above, it is possible to identify the upper and lower bounds distinguishing the two overlapping regimes as follows. Given the upper bound values for coalescence and neck breakup established above from physical arguments, the expected lower bound values for coalescence and neck breakup are $0^{\circ}$ and $60^{\circ}$, respectively. Likewise, considering the upper bound values identified for different breakup types, the lower bound values are $0^{\circ}$ for crown and disk breakups and $30^{\circ}$ for sheet breakup. The physical conditions leading to the occurrence of each raindrop collision outcome regime are summarized in Table 1.

The asymptotic case of $\mathrm{We}=0$ is attributed to coalescence rather than bounce. This behavior was explained as a result of the molecular forces by Testik (2009). For actual drop interactions, this asymptotic condition (i.e., coalescence when $\mathrm{We}=0$ ) may be extended to cover a small but finite region of the regime diagram (i.e., coalescence when $\mathrm{We} \rightarrow 0$ ). Consider two collision scenarios for two identical drops at the same terminal velocity under standard atmospheric conditions. In case I (the ideal case) the lateral drop velocities are zero, and the fall velocities are only in the vertical direction. This corresponds to a zero velocity difference, and hence $\mathrm{We}=0$. In this ideal case, the drops would stay in contact for

TABLE 1 . Summary of bounding physical conditions governing the occurrence of each identified collision outcome regime.

\begin{tabular}{lllr}
\hline \multicolumn{1}{c}{ Regime } & \multicolumn{1}{c}{$\mathrm{DE}_{1}$} & \multicolumn{1}{c}{$\mathrm{DE}_{2}$} & \multicolumn{1}{c}{$\theta$} \\
\hline Bounce & $0<\mathrm{DE}_{1}$ & $0<\mathrm{DE}_{2}<1$ & $0<\theta<90$ \\
Coalescence & $0<\mathrm{DE}_{1}<1$ & $1<\mathrm{DE}_{2}$ & $0<\theta<60$ \\
Neck breakup & $0<\mathrm{DE}_{1}$ & $1<\mathrm{DE}_{2}$ & $60<\theta<90$ \\
Sheet breakup & $1<\mathrm{DE}_{1}$ & $1<\mathrm{DE}_{2}$ & $30<\theta<60$ \\
Crown breakup & $1<\mathrm{DE}_{1}<2$ & $1<\mathrm{DE}_{2}$ & $0<\theta<30$ \\
Disk breakup & $2<\mathrm{DE}_{1}$ & $1<\mathrm{DE}_{2}$ & $0<\theta<30$ \\
\hline
\end{tabular}


TABLE 2. Probability of occurrence (\%), observed (Obs) and predicted (Pred) of each collision outcome for each region of the regime diagram. Predictions are based on the raindrop collision geometry considerations and prediction calculations are described in the text.

\begin{tabular}{|c|c|c|c|c|c|c|c|c|}
\hline \multirow[b]{3}{*}{ Collision outcome } & \multicolumn{8}{|c|}{ Region } \\
\hline & \multicolumn{2}{|c|}{ I } & \multicolumn{2}{|c|}{ IIa } & \multicolumn{2}{|c|}{ IIb } & \multicolumn{2}{|c|}{ III } \\
\hline & Obs & Pred & Obs & Pred & Obs & Pred & Obs & Pred \\
\hline Bounce (\%) & 0.6 & 0 & 0 & 0 & 0 & 0 & 71.4 & 100 \\
\hline Coalescence (\%) & 41.3 & 50 & 0 & 0 & 0 & 0 & 28.6 & 0 \\
\hline Neck $(\%)$ & 50.3 & 50 & 50.9 & 50 & 35.2 & 50 & 0 & 0 \\
\hline Sheet (\%) & 3.9 & 0 & 30.2 & 36 & 33.3 & 36 & 0 & 0 \\
\hline Disk (\%) & 0 & 0 & 0 & 0 & 31.5 & 14 & 0 & 0 \\
\hline Crown (\%) & 3.9 & 0 & 18.9 & 14 & 0 & 0 & 0 & 0 \\
\hline
\end{tabular}

enough time to coalesce rather than bounce owing to the role of cohesive molecular forces. In case II (the realistic case) the drops have lateral velocity components in addition to vertical components (see Testik et al. 2006). The difference in the lateral velocities will likely be small as compared to the vertical fall velocities (unless there are processes such as drop oscillations); hence We will be very small (recall that We is defined in terms of the absolute value of the velocity difference). As discussed above, given sufficient contact time, coalescing drops are expected for $\mathrm{We} \rightarrow 0$. However, as We increases for the collision of two identical drops (i.e., increasing lateral velocities), there may not be sufficient contact (surface and time), and hence drop bounce can occur. This scenario corresponds to large impact angles (see Fig. 5) characteristic of the so-called "grazing bounce" collisions of identical drops (i.e., $p=1$ ) reported in the literature (e.g., Qian and Law 1997). Note that terminal velocity is not a requirement for grazing bounce. That is, the model predicts that collision of any two identical drops $(p=1)$ with different velocities may result in bounce for We $<12$.

The experimental results were further independently analyzed in the context of conditional probabilities that can be used in the parameterization of raindrop collision outcomes in stochastic models of raindrop dynamics. The probabilities of occurrence of each regime for each region of the regime diagram are summarized in Table 2. This table includes both observations and predictions based on collision geometry. The predictions provided in this table involve the assumptions that (i) drops are perfectly spherical (i.e., shape deformations are absent), (ii) drops fall vertically without lateral motion, and (iii) the smaller of the two colliding drops is treated as a point (its centroid). The probability of occurrence is then predicted by simply dividing the area of the segment in the lower half of the sphere bounded by the critical impact angles proposed for a specific regime to the total area of the semisphere. Note that for the assumed fall conditions (i.e., vertical fall at terminal velocity without lateral motion) larger drops catch the smaller drops, and the collisions take place in the lower half of the larger drops. As can be seen from this table, the agreement is good, further supporting the critical impact angle values proposed in this study. Deviations for the predicted and observed occurrence probabilities are mainly limited to regions IIb and III. Region IIb corresponds to drops with high dimensionless energies (i.e., larger drops); hence, considerable drop shape deformations (i.e., flattened drop base) should be expected, which are not represented in the simple calculations described above. It is not possible to assess the deviations for the predicted and observed probabilities in region III due to the lack of robust statistics (e.g., small number of bounce observations).

\section{Discussion and conclusions}

Binary raindrop collision outcomes were investigated theoretically and experimentally. Four different drop breakup types (neck, sheet, disk, and crown breakups) are classified as subregimes. Among these breakup types, the occurrence of neck, sheet, and disk breakups was documented in previous studies; however, to our knowledge crown breakup is documented for the first time here. Although crown breakup has been classified in the past as a subcategory of disk breakup (Barros et al. 2008), its distinct morphology and fragment size distribution (observed visually) motivated its consideration as a separate breakup type in the present study. The classification of crown breakup as a separate category is further corroborated by the fact that it occupies a region of $(\mathrm{We}, p)$ space roughly between $\mathrm{DE}_{1}=1$ and $\mathrm{DE}_{1}=2$ that is clearly distinct from the remainder disk breakup collisions (Fig. 12).

The conditions for the occurrence of different subregimes in terms of bounding/critical values of the angle of impact and dimensionless energy are summarized in Table 1. An overall evaluation of the proposed regime diagram was conducted by comparing the observed 


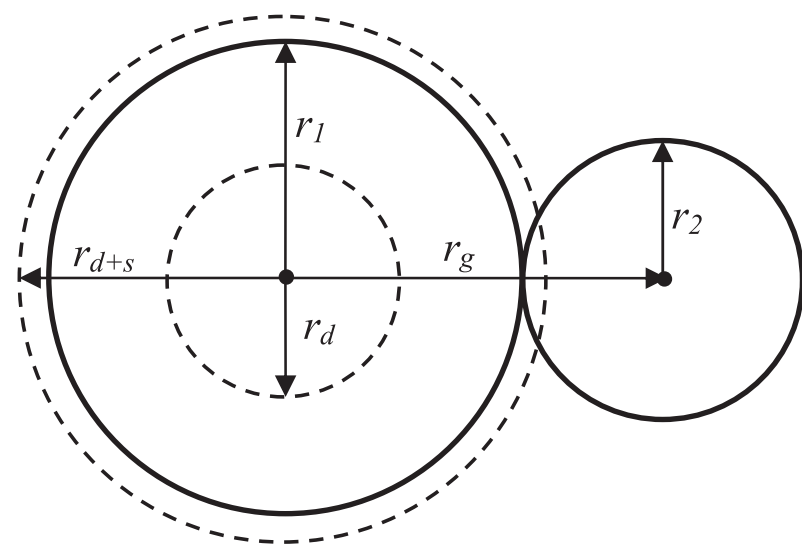

FIG. 13. Definition schematic (top view) for the data from McTaggart-Cowan and List (1975) given in Table 3. Solid circles represent colliding drops $\left[r_{1}\left(r_{2}\right)\right.$ : radius of the larger (smaller) drop]. Area bounded by the inner dashed circle (with radius $r_{d}$ ) represents the impact area for the disk breakup and the area between the outer (with radius $r_{d+s}$ ) and inner circles represents the impact area for the sheet breakup (i.e., the center of the small drop lies within the corresponding area when a sheet or disk breakup occurs). The radius of the geometric sweepout area (the sum of the radii of the small and the large drops) is denoted by $r_{g}$.

(based on NASA WFF tower experiments) and predicted (based on collision geometry considerations) probabilities of occurrence of each regime for each region of the regime diagram against each other (see Table 2), showing good agreement. The NASA WFF experiments were conducted with a single high-speed camera, and critical angles of impact were estimated indirectly as described earlier. Further verification of the regime diagram via simultaneous orthogonal highspeed camera measurements is desirable. These measurements on drop collisions are expected to be useful in determining the upper and lower bound values of angle of impact with greater accuracy and in determining if a weak functional dependency of $\theta_{\text {cr }}$ on $\mathrm{We}$ and $p$ exists.
The experimental dataset used here (Barros et al. 2008) is a laboratory dataset completely independent from that used by Testik (2009). The bench-scale drop collision laboratory data used in Testik (2009) does not replicate natural rainfall conditions (i.e., drops were not equilibrium raindrop shaped and did not fall at terminal velocity), whereas the tower data in Barros et al. emulate realistic rainfall conditions (i.e., equilibrium-shaped raindrops falling vertically at terminal velocity with minimal lateral velocity). The applicability of the We- $p$ diagram for both cases shows the generality of the proposed regime diagram. The theoretical model for collision outcome regimes is based on the dimensional analysis and considers the relative importance of the governing parameters. In doing so, the preset values of $\mathrm{DE}_{1}=1$ and $\mathrm{DE}_{2}=1$ are used to define the regime separation curves. One may expect these values to deviate from 1 due to a variety of factors (e.g., ambient factors such as pressure, temperature, relative humidity, electrical charges, viscous dissipation, etc.). The effects of these factors are not studied in our experiments to simplify the problem. Consequently, these parameters are not varied in our experiments. Nevertheless, the preset value of 1 provided a good fit to both bench-scale and tower experimental datasets. A framework for a modeling approach is presented and this approach may be used to model collision outcome regimes for drops of different fluids or to take into account the effects of the aforementioned factors. Changes and/or corrections to the preset constant may be introduced to improve accuracy, pending, however, a substantial experimental effort. An effort was made to compare the experimental results of McTaggart-Cowan and List (1975) and Qian and Law (1997) with our theoretical and experimental results as described below.

In Table 1 of McTaggart-Cowan and List (1975), ratios of the radii of impact areas for disk $r_{d}$ or disk plus sheet types $r_{d+s}$ of breakups to the radii of the larger $r_{1}$ of the colliding drops and the geometric sweepout area $r_{g}$ are given for their experimental observations. The

TABLE 3. Inferred bounding impact angle values from the experimental data of McTaggart-Cowan and List (1975). The data presented in the columns $r_{d} / r_{1}, r_{d} / r_{g}$, and $r_{d+s} / r_{1}$ (see definition schematic in Fig. 13) are reproduced from Table 1 of McTaggart-Cowan and List (1975). The values of $r_{1} / r_{g}, r_{d+s} / r_{g}$, and $r_{d} / r_{g}$ are calculated to convert the data by McTaggart-Cowan and List into the bounding impact angle values for the neck $\left[\theta_{\text {neck }}=\sin ^{-1}\left(r_{1} / r_{g}\right)\right.$, lower bound value for neck breakup $]$, sheet $\left[\theta_{\text {sheet }}=\sin ^{-1}\left(r_{d+s} / r_{g}\right)\right.$, lower bound value for sheet breakup], and disk [ $\theta_{\text {disk }}=\sin ^{-1}\left(r_{d} / r_{g}\right)$, upper bound value for disk breakup] breakups.

\begin{tabular}{|c|c|c|c|c|c|c|c|c|c|}
\hline Drop pairs & $r_{d} / r_{1}$ & $r_{d} / r_{g}$ & $r_{d+s} / r_{1}$ & $r_{1} / r_{g}$ & $r_{d+s} / r_{g}$ & $r_{d} / r_{g}$ & $\theta_{\text {neck }}\left({ }^{\circ}\right)$ & $\theta_{\text {sheet }}\left({ }^{\circ}\right)$ & $\theta_{\text {disk }}\left({ }^{\circ}\right)$ \\
\hline 4.6 and $1.8 \mathrm{~mm}$ & 0.58 & 0.42 & 1.20 & 0.72 & 0.87 & 0.42 & 46.4 & 60.3 & 24.8 \\
\hline 3.6 and $1.8 \mathrm{~mm}$ & 0.56 & 0.38 & 1.31 & 0.68 & 0.89 & 0.38 & 42.7 & 62.7 & 22.3 \\
\hline 4.6 and $1.0 \mathrm{~mm}$ & 0.55 & 0.46 & 1.03 & 0.84 & 0.86 & 0.46 & 56.7 & 59.5 & 27.4 \\
\hline 3.6 and $1.0 \mathrm{~mm}$ & 0.55 & 0.44 & 1.06 & 0.80 & 0.85 & 0.44 & 53.1 & 58.0 & 26.1 \\
\hline 3.0 and $1.0 \mathrm{~mm}$ & 0.53 & 0.39 & 1.06 & 0.74 & 0.78 & 0.39 & 47.4 & 51.2 & 23.0 \\
\hline Avg & 0.55 & 0.42 & 1.13 & 0.76 & 0.86 & 0.42 & 49.8 & 59.6 & 24.8 \\
\hline
\end{tabular}


definitions of the relevant radii are given in the schematic in Fig. 13 and the experimental data by McTaggartCowan and List (1975) are given in Table 3 for reader convenience. The impact angle values corresponding to these data are tabulated in Table 3. These calculated impact angle values indicate critical impact angle $\theta_{\text {cr }}$ values of $24.8^{\circ}$ for the upper bound of disk breakup and $59.6^{\circ}$ for the upper bound of sheet breakup. These values are remarkably close to the upper bound $\theta_{\text {cr }}$ values that we report for disk $\left(30^{\circ}\right)$ and sheet $\left(60^{\circ}\right)$ breakups. Moreover, McTaggart-Cowan and List state that, whenever neck collisions occur, the center of the smaller of the colliding drops lies outside the large drop. This qualitative statement corresponds to $\theta$ values larger than $42.7^{\circ}-56.7^{\circ}$, which again supports our proposed lower $\theta$ bound of $60^{\circ}$ for neck breakups.

Qian and Law (1997) present experimental observations for the collision of two identical water drops at $1 \mathrm{~atm}$ pressure in the nitrogen environment in Fig. 3a of their paper. Despite radically different experimental conditions (e.g., nitrogen environment, spherical drops), data presented in that figure can be compared to our results as discussed earlier. Specifically, Fig. 3a of Qian and Law shows that drops coalesce for values of the impact parameter $B$ [see geometric definition in Qian and Law (1997)] up to 0.88. This upper bound value corresponds to an impact angle value of $61^{\circ}$ compared with the $60^{\circ}$ upper bound value of the impact angle for the coalescence regime in our study. This is a remarkable agreement. Another remarkable agreement is that bounce observations were reported by Qian and Law for We values of up to approximately 12-13 (except one data point with $\mathrm{We} \approx 25$, which may well be an outlier because relative velocity and impact parameter measurement errors from photographic images may have accumulated to introduce a large error in the We number calculations), thereby further validating regions I and III of regime diagram proposed here. Qian and Law did not classify their results for different breakup regimes.

The collision outcome regime diagram proposed here provides a physical basis to improve heuristic parameterizations of raindrop collisions currently used in models of raindrop dynamics (see List and McFarquhar 1990; McFarquhar 2004; Prat and Barros 2007a,b; among others). A numerical modeling study to evaluate the benefit of using the proposed (We, $p$ ) regime diagram to describe collision outcomes is ongoing and will be presented in a forthcoming paper.

Acknowledgments. This research was supported in part by funds provided by the College of Engineering and Science at Clemson University to the first author, and NSF Grant ATM 97-530093 and NASA Grant
NNGO04GP02G to the second author. The authors are grateful to Dr. K.V. Beard and an anonymous referee for their valuable comments that have improved this manuscript significantly.

\section{REFERENCES}

Adam, J. R., N. R. Lindblad, and C. D. Hendricks, 1968: The collision, coalescence, and disruption of water droplets. J. Appl. Phys., 39, 5173-5180.

Andsager, K., K. V. Beard, and N. F. Laird, 1999: Laboratory measurements of axis ratios for large raindrops. J. Atmos. Sci., 56, 2673-2683.

Ashgriz, N., and J. Y. Poo, 1990: Coalescence and separation in binary collisions of liquid drops. J. Fluid Mech., 221, 183-204.

Barros, A. P., O. P. Prat, P. Shrestha, F. Y. Testik, and L. F. Bliven, 2008: Revisiting Low and List (1982): Evaluation of raindrop collision parameterizations using laboratory observations and modeling. J. Atmos. Sci., 65, 2983-2993.

,-- , and F. Y. Testik, 2010: Size distribution of raindrops. Nat. Phys., 6, 232.

Beard, K. V., 1977: On the acceleration of large water drops to terminal velocity. J. Appl. Meteor., 16, 1068-1071.

Blanchard, D. C., 1948: Observations on the behavior of water drops at terminal velocity in air. Occasional Rep. 7, Project Cirrus, General Electric Research Laboratory, 15 pp.

_ 1949: Experiments with water drops and the interaction between them at terminal velocity in air. Occasional Rep. 17, Project Cirrus, General Electric Research Laboratory, 29 pp.

Bliven, L. F., and T. M. Elfouhaily, 1993: Presenting the Rain-Sea Interaction Facility. NASA Ref. Pub. 1322, 51 pp.

Brazier-Smith, P. R., S. G. Jennings, and J. Latham, 1972: The interaction of falling water drops: Coalescence. Proc. Roy. Soc. London, 326A, 393-408.

Gotaas, C., P. Havelka, H. A. Jacobsen, H. F. Svendsen, M. Hase, N. Roth, and B. Weigand, 2007: Effect of viscosity on dropletdroplet collision outcome: Experimental study and numerical comparison. Phys. Fluids, 19, 102106, doi:10.1063/1.2781603.

Gunn, R., 1965: Collision characteristics of freely falling water drops. Science, 150, 695-701.

Jones, B. K., J. R. Saylor, and F. Y. Testik, 2010: Raindrop morphodynamics. Rainfall-The State of the Science, F. Y. Testik and M. Gebremichael, Eds., Amer. Geophys. Union, 7-28.

Langmuir, I., 1948: The production of rain by a chain reaction in cumulus clouds at temperatures above freezing. J. Meteor., 5, 175-192.

Lhermitte, R., 1990: Attenuation and scattering of millimeter wavelength radiation by clouds and precipitation. J. Atmos. Oceanic Technol., 7, 464-479.

List, R., and D. M. Whelpdale, 1969: A preliminary investigation of factors affecting the coalescence of colliding water drops. J. Atmos. Sci., 26, 305-308.

— and G. M. McFarquhar, 1990: The evolution of three-peak raindrop size distributions in one-dimensional shaft models. Part I: Single-pulse rain. J. Atmos. Sci., 47, 2996-3006.

— C. F. MacNeil, and J. D. McTaggart-Cowan, 1970: Laboratory investigations of temporary collisions of raindrops. J. Geophys. Res., 75, 7573-7580.

Low, T. B., and R. List, 1982a: Collision, coalescence and breakup of raindrops. Part I: Experimentally established coalescence efficiencies and fragment size distributions in breakup. J. Atmos. Sci., 39, 1591-1606. 
, and $-1982 \mathrm{~b}$ : Collision, coalescence and breakup of raindrops. Part II: Parameterization of fragment size distributions. J. Atmos. Sci., 39, 1607-1618.

Magano, C., and T. Nakamura, 1959: On the behavior of water droplets during collision with a large water drop. J. Meteor. Soc. Japan, 37, 124-127.

Magarvey, R. H., and J. W. Geldart, 1962: Drop collisions under conditions of free fall. J. Atmos. Sci., 19, 107-113.

McFarquhar, G. M., 2004: A new representation of collision-induced breakup of raindrops and its implications for the shapes of raindrop size distributions. J. Atmos. Sci., 61, 777-794.

McTaggart-Cowan, J. D., and R. List, 1975: Collision and breakup of water drops at terminal velocity. J. Atmos. Sci., 32, 1401-1411.

Menchaca-Rocha, A., F. Huidobro, A. Martinez-Davalos, K. Michaelian, A. Perez, V. Rodriguez, and N. Carjan, 1997: Coalescence and fragmentation of colliding mercury drops. J. Fluid Mech., 346, 291-318.

Montgomery, D. N., 1971: Collision and coalescence of water drops. J. Atmos. Sci., 28, 291-293.

Ochs, H. T., III, K. V. Beard, R. R. Czys, N. F. Laird, D. E. Schaufelberger, and D. J. Holdridge, 1995: Collisions between small precipitation drops. Part I: Laboratory measurements of bounce, coalescence, and temporary coalescence. J. Atmos. Sci., 52, 2258-2275.

Park, R. W., 1970: Behavior of water drops colliding in humid nitrogen. Ph.D. dissertation, University of Wisconsin, 577 pp.

Prat, O., and A. P. Barros, 2007a: A robust numerical solution of the stochastic collection-breakup equation for warm rain. J. Appl. Meteor. Climatol., 46, 1480-1497.

— characterization of microphysical processes in warm rain: Results from a homogeneous rainshaft model. Adv. Geosci., 10, 145-152. , and - 2009: Exploring the transient behavior of $Z-R$ relationships: Implications for radar rainfall estimation. J. Appl. Meteor. Climatol., 48, 2127-2143.
— , and - 2010: Assessing satellite-based precipitation estimates in the Southern Appalachian Mountains using raingauges and TRMM PR. Adv. Geosci., 25, 143-153.

Qian, J., and C. K. Law, 1997: Regimes of coalescence and separation in droplet collision. J. Fluid Mech., 331, 59-80.

Schlottke, J., W. Straub, K. D. Beheng, H. Gomass, and B. Weigand, 2010: Numerical investigation of collision-induced breakup of raindrops. Part I: Methodology and dependencies on collision energy and eccentricity. J. Atmos. Sci., 67, 557-575.

Straub, W., K. D. Beheng, A. Seifert, J. Schlottke, and B. Weigand, 2010: Numerical investigation of collision-induced breakup of raindrops. Part II: Parameterizations of coalescence efficiencies and fragment size distributions. J. Atmos. Sci., 67, 576-588.

Testik, F. Y., 2009: Outcome regimes of binary raindrop collisions. Atmos. Res., 94, 389-399.

— ture of warm rainfall: A survey. Rev. Geophys., 45, RG2003, doi:10.1029/2005RG000182.

— , and D. M. Young, 2008: Breakup patterns for binary drop collisions. J. Visualization, 11, 401-405, doi:10.1007/BF03181902.

— A. P. Barros, and L. F. Bliven, 2006: Field observations of multimode raindrop oscillations by high-speed imaging. J. Atmos. Sci., 63, 2663-2668.

Tokay, A., P. G. Bashor, E. Habib, and T. Kasparis, 2008: Raindrop size distribution measurements in tropical cyclones. Mon. Wea. Rev., 136, 1669-1685.

Villermaux, E., and B. Bossa, 2009: Single-drop fragmentation determines size distribution of raindrops. Nat. Phys., 5, 697702 .

Wang, P. K., and H. R. Pruppacher, 1977: Acceleration to terminal velocity of cloud and raindrops. J. Appl. Meteor., 16, 276-280.

Yarin, A. L., 1993: Free Liquid Jets and Films: Hydrodynamics and Rheology. Longman, $446 \mathrm{pp}$.

_ 2006: Drop impact dynamics: Splashing, spreading, receding, bouncing. ... Annu. Rev. Fluid Mech., 38, 159-192. 